\title{
Approximate Proximity Drawings
}

\author{
William Evans ${ }^{1}$, Emden R. Gansner ${ }^{2}$, Michael Kaufmann ${ }^{3}$, Giuseppe Liotta ${ }^{4}$, \\ Henk Meijer ${ }^{5}$, and Andreas Spillner ${ }^{6}$ \\ 1 University of British Columbia, Canada \\ will@cs. ubc.ca \\ 2 AT\&T Research Labs, US \\ erg@research.att.com \\ 3 Universität Tübingen, Germany \\ mkainformatik. uni-tuebingen. de \\ 4 Università degli Studi di Perugia, Italy \\ liotta@diei.unipg.it \\ 5 Roosevelt Academy, The Netherlands \\ h.meijer@roac.nl \\ 6 Universität Greifswald, Germany \\ andreas.spillner@uni-greifswald.de
}

\begin{abstract}
We introduce and study a generalization of the well-known region of influence proximity drawings, called $\left(\varepsilon_{1}, \varepsilon_{2}\right)$-proximity drawings. Intuitively, given a definition of proximity and two real numbers $\varepsilon_{1} \geq 0$ and $\varepsilon_{2} \geq 0$, an $\left(\varepsilon_{1}, \varepsilon_{2}\right)$-proximity drawing of a graph is a planar straight-line drawing $\Gamma$ such that: (i) for every pair of adjacent vertices $u, v$, their proximity region "shrunk" by the multiplicative factor $\frac{1}{1+\varepsilon_{1}}$ does not contain any vertices of $\Gamma$; (ii) for every pair of non-adjacent vertices $u, v$, their proximity region "blown-up" by the factor $\left(1+\varepsilon_{2}\right)$ contains some vertices of $\Gamma$ other than $u$ and $v$. We show that by using this generalization, we can significantly enlarge the family of the representable planar graphs for relevant definitions of proximity drawings, including Gabriel drawings, Delaunay drawings, and $\beta$-drawings, even for arbitrarily small values of $\varepsilon_{1}$ and $\varepsilon_{2}$. We also study the extremal case of $\left(0, \varepsilon_{2}\right)$-proximity drawings, which generalizes the well-known weak proximity drawing model.
\end{abstract}

\section{Introduction and Overview}

Proximity drawings are straight-line drawings of graphs where any two adjacent vertices are deemed to be close according to some proximity measure, while any two nonadjacent vertices are far from one another with respect to the same measure. Different definitions of proximity give rise to different types of proximity drawings. In the region of influence based proximity drawings two vertices $u$ and $v$ are adjacent if and only if some regions of the plane, defined by using the coordinates of $u$ and $v$, are empty, i.e. they do not contain any vertices of the drawing other than, possibly, $u$ and $v$. Throughout this paper we shall always assume that the proximity regions are closed sets; hence if a vertex is on the boundary of the proximity region of $u$ and $v$, the region is not empty.

For example, the Gabriel disk of two points $u$ and $v$ in the plane is the disk having $u$ and $v$ as their antipodal points and a Gabriel drawing (also called a Gabriel graph) is a 
planar straight-line drawing such that any two vertices are connected by an edge if and only if their Gabriel disk is empty A generalization of the Gabriel disk is the so-called $\beta$-region of influence: For a given value of $\beta$ such that $1 \leq \beta \leq \infty$, the $\beta$-region of influence of two vertices $u$ and $v$ having Euclidean distance $d(u, v)$ is the intersection of the two disks of radius $\frac{\beta d(u, v)}{2}$, centered on the line through $u$ and $v$, one containing $u$ and touching $v$, the other containing $v$ and touching $u$ (hence the $\beta$-region for $\beta=1$ is the Gabriel disk). Given a value of $\beta$, a straight-line drawing is a $\beta$-drawing (also called a $\beta$-skeleton) if and only if for any edge $(u, v)$ the $\beta$-region of influence of $u$ and $v$ is empty. Delaunay drawings use a definition of proximity that extends the one used for Gabriel drawings. Namely, the Delaunay disks of two vertices $u$ and $v$ are the disks having $\overline{u v}$ as a chord (the Gabriel disk is therefore a particular Delaunay disk). In a Delaunay drawing (also called a Delaunay graph) an edge $(u, v)$ exists if and only if at least one of the Delaunay disks of $u$ and $v$ is empty.

As is not hard to imagine, by changing the definition of region of influence, the combinatorial properties of those graphs that admit a certain type of proximity drawing can change significantly. For example, it is known that not all trees having vertices of degree four admit a Gabriel drawing [4] while they have a $\beta$-drawing for $1<\beta \leq$ $2[12]$. Unfortunately, the adoption of region of influence based proximity rules seems to dramatically restrict the family of representable graphs. Also, despite the many papers published on the topic, full combinatorial characterization of proximity drawable graphs remains an elusive goal for most types of regions of influence. The interested reader is also referred to [6[11|14] for references and results on these topics.

\subsection{Problem and Results}

In this paper, we want to compute planar straight-line drawings of graphs where adjacent vertices are relatively close to each other while non-adjacent vertices are relatively far apart. In order to overcome the restrictions on the families of representable graphs imposed by region of influence based proximity drawings, we study graph visualizations that are "good approximations" of these proximity drawings. The idea is to use slightly smaller regions of influence to justify the existence of an edge and slightly larger regions of influence to justify non-adjacent vertices.

More formally, let $D$ be a disk with center $c$ and radius $r$, and let $\varepsilon_{1}$ and $\varepsilon_{2}$ be two non-negative real numbers. The $\varepsilon_{1}$-shrunk disk of $D$ is the disk centered at $c$ and having radius $\frac{r}{1+\varepsilon_{1}}$; the $\varepsilon_{2}$-expanded disk of $D$ is the disk centered at $c$ and having radius $(1+$ $\left.\varepsilon_{2}\right) r$. An $\left(\varepsilon_{1}, \varepsilon_{2}\right)$-proximity drawing is a planar straight-line proximity drawing where the region of influence of two adjacent vertices is defined by using $\varepsilon_{1}$-shrunk disks, while the region of influence of two non-adjacent vertices uses $\varepsilon_{2}$-expanded disks. In the next sections we study $\left(\varepsilon_{1}, \varepsilon_{2}\right)$-Gabriel drawings, $\left(\varepsilon_{1}, \varepsilon_{2}\right)$-Delaunay drawings, and $\left(\varepsilon_{1}, \varepsilon_{2}\right)$ - $\beta$-drawings.

It is immediate to observe that all planar graphs with at least one edge or at least three vertices have an $\left(\varepsilon_{1}, \varepsilon_{2}\right)$-proximity drawing for sufficiently large values of $\varepsilon_{1}, \varepsilon_{2}$. For example, every planar straight-line drawing $\Gamma$ with at least one edge is a $(\infty, \infty)$ Gabriel drawing since an $\infty$-shrunk Gabriel disk reduces to a point (and thus the $\infty$ shrunk disk of every edge in $\Gamma$ is empty) and an $\infty$-expanded Gabriel disk is the whole plane (and thus the $\infty$-expanded disk of every pair of non-adjacent vertices of $\Gamma$ is never 
empty). At the other extreme, a (0,0)-Gabriel drawing is a Gabriel drawing, since a 0shrunk Gabriel disk is a Gabriel disk and so is a 0-expanded Gabriel disk. Hence, not all planar graphs admit a (0,0)-Gabriel drawing [4].

Based on this observation, our main target is to establish values of $\varepsilon_{1}$ and of $\varepsilon_{2}$ that make it possible to compute $\left(\varepsilon_{1}, \varepsilon_{2}\right)$-proximity drawings for meaningful families of planar graphs. Our results are as follows:

- We prove that every embedded planar graph admits, for any $\varepsilon_{1}>0$ and any $\varepsilon_{2}>$ 0 , an $\left(\varepsilon_{1}, \varepsilon_{2}\right)$-Gabriel drawing, an $\left(\varepsilon_{1}, \varepsilon_{2}\right)$-Delaunay drawing, and an $\left(\varepsilon_{1}, \varepsilon_{2}\right)-\beta$ drawing (for all $1 \leq \beta \leq \infty$ ) that preserve the given embedding. (See Theorems 1 4 and 5)

- We show that the above results are, in a sense, tight by exhibiting embedded planar graphs that do not have an embedding preserving $\left(\varepsilon_{1}, \varepsilon_{2}\right)$-proximity drawing with either $\varepsilon_{1}=0$ or $\varepsilon_{2}=0$. (See again Theorems 1, 4, and5)

- We study $\left(0, \varepsilon_{2}\right)$-proximity drawings which, as explained in the next section, make it possible to express different proximity conventions in a unified framework. In particular, we study $\left(0, \varepsilon_{2}\right)$-Gabriel drawings of outerplanar graphs, extending previous results of [7,12]. (See Theorems 2 and 3 )

We emphasize that the main contribution of this paper is in introducing the concept of $\left(\varepsilon_{1}, \varepsilon_{2}\right)$-proximity drawing and in proving the existence of $\left(\varepsilon_{1}, \varepsilon_{2}\right)$-proximity drawings for relevant families of graphs. Hence, we shall not spend words on the time complexities of our algorithms; it is not hard to see, however, that our drawing techniques all require polynomial time when adopting the real RAM model of computation. For reasons of space, some proofs are sketched or omitted.

\subsection{Related Work}

Several generalizations, variants, and relaxations of proximity drawings have been defined in the literature such as, for example, $k$-localized Delaunay triangulations, approximate minimum spanning trees, and witness proximity drawings. The interested reader can, for example, use [2:10 13] as starting points to study these topics.

Although each proximity drawing mentioned above would deserve some special attention, in this introduction we can just spend a few words on weak proximity drawings [7] that are more closely related with $\left(\varepsilon_{1}, \varepsilon_{2}\right)$-proximity drawings. In a weak proximity drawing, the region of influence of any pair of adjacent vertices must be empty, while no condition is given for the non-adjacent pairs. Hence, weak proximity drawings guarantee visual closeness of groups of edge-related vertices but do not ensure that unrelated vertices are far apart. In contrast, $\left(\varepsilon_{1}, \varepsilon_{2}\right)$-proximity drawings guarantee some relative closeness of the adjacent pairs of vertices and some relative separation of the non-adjacent pairs for any finite values of $\varepsilon_{1}$ and $\varepsilon_{2}$.

Note that a weak proximity drawing is a $(0, \infty)$-proximity drawing and that a proximity drawing in the traditional sense is a $(0,0)$-proximity drawing. Therefore, $\left(0, \varepsilon_{2}\right)$-proximity drawings make it possible to study proximity drawability in a unified framework: as the value of $\varepsilon_{2}$ increases, $\left(0, \varepsilon_{2}\right)$-proximity drawings approach weak proximity drawings. Several questions can be asked within this unifying framework. 
For example, not all trees have a Gabriel drawing, while all trees have a weak Gabriel drawing. What is the minimum threshold value such that if $\varepsilon_{2}$ is larger than this threshold all trees are drawable? Theorem 2 answers this question.

\section{Approximate Gabriel Drawings}

Let $\Gamma$ be a planar straight-line drawing of a graph and let $\varepsilon_{1}, \varepsilon_{2}$ be two non-negative numbers. Let $u, v$ be any two vertices of $\Gamma$ and let $D(u, v)$ be the Gabriel disk of $u, v$ (that is, the disk having $u$ and $v$ as the end-points of its diameter). We say that $\Gamma$ is an $\left(\varepsilon_{1}, \varepsilon_{2}\right)$-Gabriel drawing if: (i) for every edge $(u, v)$ of $\Gamma$ the $\varepsilon_{1}$-shrunk disk of $D(u, v)$ is empty (i.e. it does not contain any vertex of $\Gamma$ other than, possibly, $u$ and $v$ ); and (ii) for every pair of non-adjacent vertices $u, v$ of $\Gamma$, the $\varepsilon_{2}$-expanded disk of $D(u, v)$ is not empty (i.e. it contains some vertex $w$ of $\Gamma$ other than $u$ and $v$ ). Note that a Gabriel graph is a special case of an $\left(\varepsilon_{1}, \varepsilon_{2}\right)$-Gabriel drawing, namely the one in which $\varepsilon_{1}=\varepsilon_{2}=0$.

Fig. 1 is an example of an $\left(\varepsilon_{1}, \varepsilon_{2}\right)$-Gabriel drawing for $\varepsilon_{1}=0$ and $\varepsilon_{2}=0.7$. The drawing is not a Gabriel drawing; for example, the dotted disk in the figure is a Gabriel disk, while the solid one is its 0.7-expanded version. Note that no Gabriel drawing exists for the tree in Fig. 1] [4].

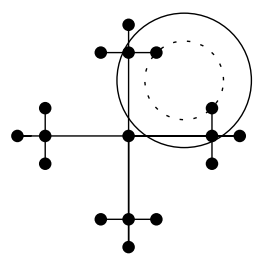

Fig. 1. A (0,0.7)-Gabriel drawing of a tree that does not have a $(0,0)$-Gabriel drawing

In order to establish values of $\varepsilon_{1}, \varepsilon_{2}$ that allow an $\left(\varepsilon_{1}, \varepsilon_{2}\right)$-Gabriel drawing of every planar graph, we start by considering the extremal cases that either $\varepsilon_{1}=0$ and $\varepsilon_{2}>0$ or $\varepsilon_{1}>0$ and $\varepsilon_{2}=0$. An embedded planar graph is a planar graph together with a planar topological embedding. A planar straight-line drawing $\Gamma$ of an embedded planar graph $G$ maintains (or preserves) the embedding of $G$ if $\Gamma$ and $G$ have the same set of faces and for every vertex $v$ the circular order of the edges around $v$ is the same in $G$ and in $\Gamma$; in this case we shall also sometimes say that $\Gamma$ is an embedding preserving drawing of $G$.

The next two lemmas study the relationship between embedding preserving $\left(\varepsilon_{1}, \varepsilon_{2}\right)$ Gabriel drawings with either $\varepsilon_{1}=0$ or $\varepsilon_{2}=0$ and Gabriel graphs. We say that an embedded planar graph is Gabriel drawable if there exists a Gabriel graph $\Gamma$ such that $\Gamma$ is an embedding preserving drawing of $G$.

Lemma 1. Let $G$ be an embedded maximal planar graph, that is, a triangulation, and let $\varepsilon_{2}$ be any given real number such that $\varepsilon_{2} \geq 0$. G has an embedding preserving $\left(0, \varepsilon_{2}\right)$-Gabriel drawing if and only if $G$ has an embedding preserving Gabriel drawing.

Proof. If $G$ has an embedding preserving Gabriel drawing $\Gamma$, then $\Gamma$ is also a $(0,0)$ Gabriel drawing of $G$ and therefore a $\left(0, \varepsilon_{2}\right)$-Gabriel drawing for any $\varepsilon_{2}>0$. If $G$ has an embedding preserving $\left(0, \varepsilon_{2}\right)$-Gabriel drawing $\Gamma$, let $V$ be the vertex set of $\Gamma$. Let $G G(V)$ be the Gabriel graph having $V$ as its vertex set. Since $\Gamma$ is a $\left(0, \varepsilon_{2}\right)$-Gabriel drawing, for every pair $u, v$ of adjacent vertices in $\Gamma$ the disk having $u$ and $v$ as antipodal points does not contain any other element of $V$. Hence, every edge of $\Gamma$ is also an edge in $G G(V)$. Since the Gabriel graph of a point set is a planar geometric graph [11] and $G$ is a triangulation, it follows that $G G(V)$ coincides with $\Gamma$. 
It is immediate to verify that every embedded planar triangulation with a separating three-cycle does not have a Gabriel drawing. Therefore, Lemma1 1 implies the following.

Corollary 1. There exist embedded planar graphs that do not have an embedding preserving $\left(0, \varepsilon_{2}\right)$-Gabriel drawing, for any $\varepsilon_{2} \geq 0$.

The proof of the next lemma is omitted. It can be established using a similar argument to the one in the proof of Lemma 1 , but focusing on pairs of non-adjacent vertices. The key observation is that, for any embedding preserving $\left(\varepsilon_{1}, 0\right)$-Gabriel drawing $\Gamma$ of an embedded tree $T$, any edge of the Gabriel graph $G G(V)$ of the points in the vertex set $V$ of $\Gamma$ must also be an edge of $\Gamma$. But this implies, since $G G(V)$ is connected [11], that $G G(V)$ coincides with $\Gamma$.

Lemma 2. Let $T$ be an embedded tree and let $\varepsilon_{1}$ be any given real number such that $\varepsilon_{1} \geq 0$. T has an embedding preserving $\left(\varepsilon_{1}, 0\right)$-Gabriel drawing if and only if $T$ has an embedding preserving Gabriel drawing.

Lemma 2 and the characterization of which trees admit a Gabriel drawing in [4] immediately imply the following.

Corollary 2. There exist embedded planar graphs that do not have an embedding preserving $\left(\varepsilon_{1}, 0\right)$-Gabriel drawing, for any $\varepsilon_{1} \geq 0$.

Motivated by Corollaries 1 and 2 , we move our attention to $\left(\varepsilon_{1}, \varepsilon_{2}\right)$-Gabriel drawings where both $\varepsilon_{1}>0$ and $\varepsilon_{2}>0$. We prove that one can compute a drawing that approximates a Gabriel drawing for (almost) every planar graph, provided that the Gabriel region is scaled down for the edges and is scaled up for the non-adjacent pairs of vertices by any arbitrarily small chosen amount. Note, however, that for small values of $\varepsilon_{2}$ any $\left(\varepsilon_{1}, \varepsilon_{2}\right)$-Gabriel drawing must contain at least one edge, namely between a pair of vertices with minimum distance.

Lemma 3. Let $\varepsilon_{1}, \varepsilon_{2}$ be any two real numbers such that $\varepsilon_{1}>0$ and $\varepsilon_{2}>0$. Every embedded planar graph with at least one edge has an embedding preserving $\left(\varepsilon_{1}, \varepsilon_{2}\right)$ Gabriel drawing.

Proof. Let $G$ be a planar graph with a given planar embedding. Consider some maximal planar supergraph $G^{\prime}$ of $G$ together with a planar embedding of $G^{\prime}$ that respects the given embedding of $G$. We choose $G^{\prime}$ in such a way that there is a canonical ordering $v_{1}, \ldots, v_{n}$ of the vertices of $G^{\prime}$ so that $\left(v_{1}, v_{2}\right)$ is an edge of $G$. Let $G_{i}$ be the subgraph of $G$ induced by $V_{i}=\left\{v_{1}, v_{2}, \ldots, v_{i}\right\}$. We show how to construct a drawing $\Gamma_{i}$ of $G_{i}$ by induction so that, for all $i \geq 2$, (a) $\Gamma_{i}$ is an embedding preserving $\left(\varepsilon_{1}, \varepsilon_{2}\right)$-Gabriel drawing of $G_{i}$; (b) all vertices in $V_{i}$ that lie on the outer face of $\Gamma_{i}$ are horizontally visible from the right; and (c) vertices $v_{2}, v_{3}, \ldots, v_{i}$ have $y$-coordinates $n, n-1, \ldots, n-i+2$. Clearly we can satisfy these properties for $i=2$, since $\left(v_{1}, v_{2}\right)$ is an edge of $G$, by drawing $v_{1}$ and $v_{2}$ at points $(0,1)$ and $(0, n)$, respectively.

Next, assuming we have $\Gamma_{i}$ for some $i \geq 2$, we show how to construct $\Gamma_{i+1}$. We will place vertex $v_{i+1}$ at $y$-coordinate $n-i+1$ far enough to the right so that for every $v_{j}, v_{k} \in V_{i}$, (i) an edge from $v_{i+1}$ to $v_{j}$ is permitted by the $\varepsilon_{1}$-shrunk Gabriel disk 
$D\left(v_{i+1}, v_{j}\right)$ (i.e., the shrunken disk is empty); (ii) if $\left(v_{i+1}, v_{j}\right)$ is not an edge in $G$ then the $\varepsilon_{2}$-expanded Gabriel disk $D\left(v_{i+1}, v_{j}\right)$ prevents the edge (i.e., the expanded disk contains a vertex); and (iii) $v_{i+1}$ does not lie in the $\varepsilon_{1}$-shrunk Gabriel disk $D\left(v_{j}, v_{k}\right)$.

Let $D$ be the smallest disk centered on $y$-coordinate $n-i+1$ that encloses $\Gamma_{i}$. Let $c$ be the center of $D$ and $r$ be the radius of $D$. Let $\ell r$ be the (still to be determined) distance of $v_{i+1}$ from the rightmost point of $D$. We choose $\ell$ so that for every $p \in D$, if $C$ is the disk with diameter $\overline{p v_{i+1}}$ (in fact, $C$ can be any disk with chord $\overline{p v_{i+1}}$ ), (I) the $\varepsilon_{1}$-shrunk $C$ does not intersect $D$ (implying Property (i)), and (II) the $\varepsilon_{2}$-expanded $C$ contains $D$ (implying Property (ii)). Let $b$ be the center of $C$. Since $b$ is on the perpendicular bisector of $\overline{p v_{i+1}}, d(b, p) \geq(\ell / 2) r$. Refer to Fig. 2.

Property (I) is equivalent to $\frac{d(b, p)}{d(b, c)-r}<1+\varepsilon_{1}$. By the triangle inequality, $d(b, p) \leq d(b, c)+$ $d(c, p) \leq d(b, c)+r$. Thus, $\frac{d(b, p)}{d(b, c)-r} \leq \frac{d(b, p)}{d(b, p)-2 r}$ $=1+\frac{2 r}{d(b, p)-2 r} \leq 1+\frac{2 r}{(\ell / 2) r-2 r}=1+\frac{4}{\ell-4}$. Property (II) is equivalent to $\frac{d(b, c)+r}{d(b, p)}<1+\varepsilon_{2}$. By the triangle inequality, $d(b, c) \leq d(b, p)+$ $d(p, c) \leq d(b, p)+r$. Thus, $\frac{d(b, c)+r}{d(b, p)} \leq \frac{d(b, p)+2 r}{d(b, p)}$ $=1+\frac{2 r}{d(b, p)} \leq 1+4 / \ell$. If we choose $\ell$ large enough so that $4 /(\ell-4)<\varepsilon_{1}$ and $4 / \ell<\varepsilon_{2}$ then we satisfy both Properties (i) and (ii). Property (iii) is immediate since the $\varepsilon_{1}$-shrunk disks $D\left(v_{j}, v_{k}\right)$ for edges $\left(v_{j}, v_{k}\right)$ with $v_{j}, v_{k} \in V_{i}$ are

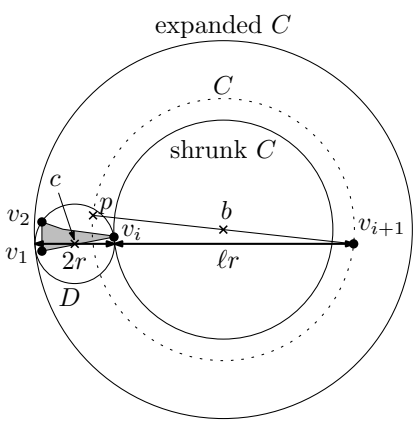

Fig. 2. Proof of Lemma 3 contained in $D$ and $v_{i+1}$ lies outside $D$. In addition, we choose $\ell$ large enough so that no edge from $v_{i+1}$ to $v_{j}, j \leq i$, crosses any (already drawn) edge in $\Gamma_{i}$. Thus, we ensure that $\Gamma_{i+1}$ respects the given embedding and is a $\left(\varepsilon_{1}, \varepsilon_{2}\right)$-Gabriel drawing of $G_{i+1}$.

The results in this section can be summarized as follows.

Theorem 1. Let $G$ be an embedded planar graph with at least one edge. For any given values of $\varepsilon_{1}, \varepsilon_{2}$ such that $\varepsilon_{1}>0$ and $\varepsilon_{2}>0, G$ admits an embedding preserving $\left(\varepsilon_{1}, \varepsilon_{2}\right)$-Gabriel. Also, there exist embedded planar graphs that do not have an embedding preserving $\left(0, \varepsilon_{2}\right)$-Gabriel drawing and embedded planar graphs that do not have an embedding preserving $\left(\varepsilon_{1}, 0\right)$-Gabriel drawing.

Theorem 1 naturally gives rise to two research directions. One is about extending the set of proximity regions that make it possible to compute $\left(\varepsilon_{1}, \varepsilon_{2}\right)$-proximity drawings for all planar graphs and for any arbitrarily small positive values of $\varepsilon_{1}$ and $\varepsilon_{2}$. The second is about studying subfamilies of planar graphs that admit an $\left(\varepsilon_{1}, \varepsilon_{2}\right)$-Gabriel drawing in the extremal cases that either $\varepsilon_{1}=0$ or $\varepsilon_{2}=0$. The next two sections study these questions; as for the extremal case, we shall focus on $\left(0, \varepsilon_{2}\right)$-Gabriel drawings because, as explained in the introduction, they generalize the notion of weak Gabriel drawings. 


\section{$3\left(0, \varepsilon_{2}\right)$-Gabriel Drawings}

This section studies $\left(0, \varepsilon_{2}\right)$-Gabriel drawings. Observe that this family of approximate proximity drawings generalizes weak Gabriel drawings, which are equivalent to $(0, \infty)$-Gabriel drawings. Di Battista et al. [7] proved that all biconnected outerplanar graphs and all trees have a $(0, \infty)$-Gabriel drawing, while Bose et al. [4] proved that not all trees have a $(0,0)$-Gabriel drawing. The next two lemmas and Theorem 2 establish a tight threshold value for $\varepsilon_{2}$ for the $\left(0, \varepsilon_{2}\right)$-Gabriel drawability of embedded trees.

Lemma 4. For any real number $\varepsilon_{2}<2$, there exists a tree that does not admit a $\left(0, \varepsilon_{2}\right)$ Gabriel drawing.

Proof. Let $0 \leq \varepsilon_{2}<2$ be a real number. Consider the star tree $S_{d}$ with central vertex $v$ of degree $d$. We show that if $d$ is sufficiently large then $S_{d}$ has no $\left(0, \varepsilon_{2}\right)$-Gabriel drawing. To this end, consider an arbitrary drawing $\Gamma$ of $S_{d}$ and assume for a contradiction that $\Gamma$ is a $\left(0, \varepsilon_{2}\right)$-Gabriel drawing. Select two distinct leaves $u$ and $w$ of $S_{d}$ such that in $\Gamma$ the angle $\alpha$ between $\overline{u v}$ and $\overline{v w}$ is minimal. Note that, for $d$ sufficiently large, we have $\alpha<\pi / 4$.

We assume without loss of generality that $d(v, w) \leq d(u, v)=1$ holds. Let $c$ denote the midpoint of $\overline{u w}$. The situation is depicted in Fig. 3 Note that, since $D(u, v)$ does not contain any vertices other than $u$ and $v$, we have $d(v, w) \geq \cos \alpha$. This implies $\sin \alpha \leq d(u, w) \leq 2 \sin \frac{\alpha}{2}$.

Since $\alpha$ is minimal, the shaded area in Fig. 3, that is, the wedges with apex $v$ and aperture angle $\alpha$ adjacent to the

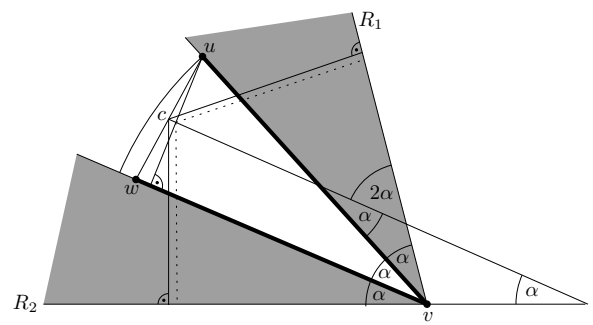

Fig. 3. Proof of Lemma 4 wedge defined by $u, v$ and $w$, cannot contain any vertex in their interior. Hence, to obtain a lower bound on the minimum value by which $D(u, w)$ must be expanded to contain a vertex other than $u$ and $w$, it suffices to consider the minimum of $\frac{2 d(c, v)}{d(u, w)}, \frac{2 d_{1}}{d(u, w)}$, and $\frac{2 d_{2}}{d(u, w)}$, where $d_{1}$ and $d_{2}$ denote the distance of $c$ from the rays $R_{1}$ and $R_{2}$, respectively (see Fig. 3).

Now, we have $\frac{2 d(c, v)}{d(u, w)} \geq \frac{\cos \alpha}{\sin (\alpha / 2)}$ which tends to $+\infty$ as $d$ tends to $+\infty$ and, thus, $\alpha$ tends to 0 . It also follows, using simple geometric arguments, that

$$
\begin{aligned}
& \frac{2 d_{1}}{d(u, w)} \geq\left(\frac{\cos \alpha}{2}+\frac{1}{4 \cos \alpha}\right) \frac{\sin (2 \alpha)}{\sin (\alpha / 2)} \text { and } \\
& \frac{2 d_{2}}{d(u, w)} \geq\left(\frac{\cos \alpha}{2}+\frac{1}{4 \cos \alpha}+\frac{\cos (2 \alpha)}{4 \cos \alpha}+\frac{\sin (2 \alpha)}{4 \sin \alpha}\right) \frac{\sin \alpha}{\sin (\alpha / 2)}
\end{aligned}
$$

hold. It is routine to check that the right hand sight in both inequalities above tends to 3 as $d$ tends to $+\infty$. But this implies that, for sufficiently large $d$, the $\varepsilon_{2}$-expanded disk $D(u, w)$ does not contain any vertices other than $u$ and $w$, a contradiction. 
Lemma 5. Let $T$ be an embedded tree. Then $T$ admits an embedding preserving $\left(0, \varepsilon_{2}\right)$ -Gabriel drawing for any real number $\varepsilon_{2} \geq 2$.

Proof. (Sketch) Root $T$ at an arbitrary vertex $t$. First, draw $t$ at an arbitrary point. In general, let $W$ and $E(W)$ denote the set of vertices and edges, respectively, already drawn. In a single step of our algorithm we consider an arbitrary $v \in W$ such that the set $U$ of its children are all undrawn. If no such $v$ exists, we will have drawn $T$.

Let $r$ be the minimum of $\frac{1}{2} \min \{d(v, w): w \in W \backslash\{v\}\}$ and $\frac{1}{2} \min \left\{d\left(v, D\left(w, w^{\prime}\right)\right)\right.$ : $\left(w, w^{\prime}\right) \in E(W)$ and $\left.w, w^{\prime} \neq v\right\}$, where $d(v, D)$ is the distance from $v$ to disk $D$ (if $v$ is $t$ then set $r=1$ ). Draw the vertices in $U$ equally spaced on the semicircle of radius $r$ centered at $v$ whose base side is perpendicular to the line segment between $v$ and $v$ 's parent, so that the given embedding of $T$ is maintained (cf. Fig. 4 (a) where the semicircle is drawn shaded).

Note that, for all $u \in U$, (a) the disk $D(v, u)$ is empty, (b) the $\varepsilon_{2}$-expanded disk of $D(u, w)$ contains $v$ for all $w \in W \backslash\{v\}$, (c) the edge $(v, u)$ does not cross any edge in $E(W)$, and (d) for every edge $\left(w, w^{\prime}\right) \in$ $E(W), D\left(w, w^{\prime}\right)$ does not contain $u$.

It remains to show that, for every pair $u, u^{\prime} \in U, u \neq$ $u^{\prime}$, the $\varepsilon_{2}$-expanded disk of $D\left(u, u^{\prime}\right)$, denoted in the following by $D^{\prime}$, contains a vertex in $W \cup U \backslash\left\{u, u^{\prime}\right\}$. If $u$ and $u^{\prime}$ are not consecutive on the semicircle then a vertex in $U$ between them lies in $D^{\prime}$. Otherwise, let $\alpha=\angle u v u^{\prime}$. If $\alpha \geq \pi / 4$ then $v$ is in $D^{\prime}$. If $\alpha \leq \pi / 5$ then the vertex $u^{\prime \prime} \in U$ that follows $u^{\prime}$ is in $D$ (see Fig.4(b)).

(a)

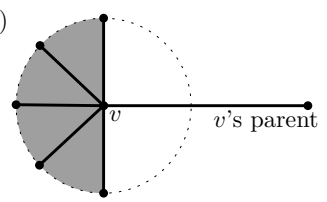

(b)

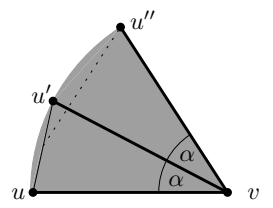

Fig. 4. Proof of Lemma5

Lemmas 4 and 5 can be summarized as follows.

Theorem 2. Every embedded tree has an embedding preserving $\left(0, \varepsilon_{2}\right)$-Gabriel drawing for any given value of $\varepsilon_{2}$ such that $\varepsilon_{2} \geq 2$. Also, for each value of $\varepsilon_{2}$ such that $0 \leq \varepsilon_{2}<2$, there exists a tree $T$ such that $T$ does not have a $\left(0, \varepsilon_{2}\right)$-Gabriel drawing.

We now consider outerplanar graphs with cycles. Lenhart and Liotta [12] proved that all biconnected outerplanar graphs with a given outerplanar embedding have a $(0,0)$ Gabriel drawing that maintains the embedding, while a connected outerplanar graph where a cut vertex is shared by more that four biconnected components is not $(0,0)$ Gabriel drawable. The next theorem shows that this upper bound on the number of components sharing a cutvertex can be removed in $\left(0, \varepsilon_{2}\right)$-Gabriel drawings, provided that the input graph does not have any degree-one vertices. In the statement, by embedded outerplanar graph we mean an outerplanar graph with a planar embedding where all vertices are on the external face. The proof is omitted.

Theorem 3. Let $G$ be an embedded outerplanar graph that does not have vertices of degree one or zero. $G$ has a $\left(0, \varepsilon_{2}\right)$-Gabriel drawing that maintains the embedding for any given value of $\varepsilon_{2}$ such that $\varepsilon_{2}>0$. 


\section{Approximate $\beta$-Drawings and Delaunay Drawings}

In this section we extend Theorem 1 to other families of $\left(\varepsilon_{1}, \varepsilon_{2}\right)$-proximity drawings. Subsection 4.1 studies an infinite family of $\left(\varepsilon_{1}, \varepsilon_{2}\right)$-proximity drawings that includes the $\left(\varepsilon_{1}, \varepsilon_{2}\right)$-Gabriel drawings as a special case. Subsection 4.2 introduces and studies approximations of the Delaunay drawings.

\section{$4.1\left(\varepsilon_{1}, \varepsilon_{2}\right)-\beta$-Drawings}

Let $\varepsilon_{1}, \varepsilon_{2}$ be any two non-negative numbers and let $\beta$ be any real number such that $\beta \geq 1$. Let $\Gamma$ be a planar straight-line drawing of a graph and let $u, v$ be any two vertices of $\Gamma$. The $\beta$-region of influence of $u$ and $v$, denoted as $\beta(u, v)$, is the intersection of two disks $D_{u}$ and $D_{v}$ such that: (i) both $D_{u}$ and $D_{v}$ have the center along the line through $u, v$; (ii) both $D_{u}$ and $D_{v}$ have radius $\frac{\beta d(u, v)}{2}$, where $d(u, v)$ is the Euclidean distance between $u$ and $v ; D_{u}$ contains $v$ and $D_{v}$ contains $u$; and (iii) the circumference of $D_{u}$ contains $u$ and the circumference of $D_{v}$ contains $v$. The $\varepsilon_{1}$-shrunk $\beta$-region of influence of $u$ and $v$ is defined as the intersection of the $\varepsilon_{1}$-shrunk disk of $D_{u}$ with the $\varepsilon_{1}$-shrunk disk of $D_{v}$. Similarly, the $\varepsilon_{2}$-expanded $\beta$-region of influence of $u$ and $v$ is the intersection of the $\varepsilon_{2}$-expanded disks of $D_{u}$ and $D_{v}$.

We say that $\Gamma$ is an $\left(\varepsilon_{1}, \varepsilon_{2}\right)-\beta$-drawing if: (i) for every edge $(u, v)$ of $\Gamma$ the $\varepsilon_{1}$-shrunk $\beta$-region of influence of $u$ and $v$ is empty; and (ii) for every pair of non-adjacent vertices $u, v$ of $\Gamma$, the $\varepsilon_{2}$-expanded $\beta$-region of influence of $u$ and $v$ is not empty.

Not all embedded planar graphs have a $(0,0)-\beta$-drawing [4]. Also, by definition, an $\left(\varepsilon_{1}, \varepsilon_{2}\right)$ - $\beta$-drawing with $\beta=1$ is a $\left(\varepsilon_{1}, \varepsilon_{2}\right)$-Gabriel drawing. Hence, by Corollaries 1 and 2, it follows that not all embedded planar graphs admit an $\left(\varepsilon_{1}, \varepsilon_{2}\right)-\beta$-drawing that respects the given embedding, when either $\varepsilon_{1}$ or $\varepsilon_{2}$ is set to 0 . On the other hand, we can extend Lemma 3 to all values of $\beta>1$. The proof technique is similar to the one in Lemma 3 Therefore, the proof is omitted.

Lemma 6. Let $\varepsilon_{1}, \varepsilon_{2}$ be any two real numbers such that $\varepsilon_{1}>0$ and $\varepsilon_{2}>0$ and let $\beta$ be any real number such that $\beta \geq 1$. Every embedded planar graph with at least one edge has a $\left(\varepsilon_{1}, \varepsilon_{2}\right)$ - $\beta$-drawing that maintains the given embedding.

We can summarize the discussion of this section as follows.

Theorem 4. Let $G$ be an embedded planar graph with at least one edge. For any given values of $\varepsilon_{1}, \varepsilon_{2}$ such that $\varepsilon_{1}>0$ and $\varepsilon_{2}>0$ and for any value of $\beta$ such that $\beta \geq 1$, $G$ admits an embedded $\left(\varepsilon_{1}, \varepsilon_{2}\right)$ - $\beta$-drawing. Also, there exist embedded planar graphs that do not have a $\left(0, \varepsilon_{2}\right)$ - $\beta$-drawing and planar graphs that do not have a $\left(\varepsilon_{1}, 0\right)-\beta$ drawing that maintain the given embedding.

\section{$4.2\left(\varepsilon_{1}, \varepsilon_{2}\right)$-Delaunay drawings}

Let $\Gamma$ be a planar straight-line drawing of a graph and let $\varepsilon_{1}, \varepsilon_{2}$ be any two non-negative numbers. Let $u, v$ be any two vertices of $\Gamma$ and let $\mathcal{D}(u, v)$ be the set of all disks in the plane that have $\overline{u v}$ as a chord. Let $\mathcal{D}_{\varepsilon_{1}}(u, v)$ be the set of the $\varepsilon_{1}$-shrunk disks of 
$\mathcal{D}(u, v)$ and let $\mathcal{D}_{\varepsilon_{2}}(u, v)$ be the set of the $\varepsilon_{2}$-expanded disks of $\mathcal{D}(u, v)$. The drawing $\Gamma$ is an $\left(\varepsilon_{1}, \varepsilon_{2}\right)$-Delaunay drawing if: (i) for any two adjacent vertices $u, v$ of $\Gamma$, there exists at least one empty disk in $\mathcal{D}_{\varepsilon_{1}}(u, v)$; and (ii) for any two non-adjacent vertices $u, v$ of $\Gamma$, all disks of $\mathcal{D}_{\varepsilon_{2}}(u, v)$ contain some vertex of $\Gamma$ other than $u$ and $v$. Note that a Delaunay drawing is a special case of $\left(\varepsilon_{1}, \varepsilon_{2}\right)$-Delaunay drawings, namely the one in which $\varepsilon_{1}=\varepsilon_{2}=0$. Fig. 5 is an example of an $\left(\varepsilon_{1}, \varepsilon_{2}\right)$-Delaunay drawing for $\varepsilon_{1}=0.25$ and $\varepsilon_{2}=0.2$. In this figure, two Delaunay disks are described (dotted) and their corresponding $\varepsilon_{1}$-shrunk and $\varepsilon_{2}$-expanded counterparts (solid) are depicted. The graph with the planar embedding of Fig. 5 does not admit an embedding preserving Delaunay drawing [8].

Recall that, in the context of Delaunay drawings, a point set $P$ is degenerate if either four or more co-circular points in $P$ define a circle that does not contain another point in $P$ in its interior, or there are three or more collinear points in $P$ on the boundary of the convex hull of $P$ (see, e.g. [9]). Note that, for any $(0,0)$-Delaunay drawing $\Gamma$, if the point set $P$ representing the vertices in $\Gamma$ is non-degenerate, $\Gamma$ coincides with the well-known non-degenerate Delaunay triangulation of $P$. We say that an embedded maximal planar graph $G$ is Delaunay drawable if the exists a set of points such that the

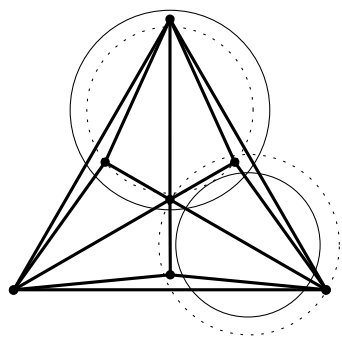

Fig. 5. An $\left(\varepsilon_{1}, \varepsilon_{2}\right)$-Delaunay drawing for $\varepsilon_{1}=0.25$ and $\varepsilon_{2}=0.2$ of a planar embedded graph that does not have an embedding preserving Delaunay drawing Delaunay graph of this point set is an embedding preserving drawing of $G$. Since Delaunay triangulations are among the most studied graphs in computational geometry, we start by investigating the relationship between $\left(\varepsilon_{1}, \varepsilon_{2}\right)$-Delaunay drawability and Delaunay drawability of maximal planar graphs. The proof of the next lemma is again omitted.

Lemma 7. There exist embedded maximal planar graphs that do not admit an embedding preserving $\left(\varepsilon_{1}, 0\right)$-Delaunay drawing, for any value $\varepsilon_{1} \geq 0$.

While Lemma 7 considers $\left(\varepsilon_{1}, 0\right)$-Delaunay drawings of maximal planar triangulations, one can wonder what happens with the other extreme, that is, with $\left(0, \varepsilon_{2}\right)$-Delaunay drawings. With arguments similar to those in the proof of Lemma 1 and the results in [9], the following lemma and corollary can be proved.

Lemma 8. Let $G$ be an embedded maximal planar triangulation and let $\varepsilon_{2}$ be any given real number such that $\varepsilon_{2} \geq 0$. $G$ has an embedding preserving $\left(0, \varepsilon_{2}\right)$-Delaunay drawing if and only if $G$ is Delaunay drawable.

Corollary 3. There exist embedded planar graphs that do not have an embedding preserving $\left(0, \varepsilon_{2}\right)$-Delaunay drawing, for any $\varepsilon_{2} \geq 0$.

By using again similar arguments as those in the proof of Lemma 3 we can prove the following. 
Lemma 9. Let $\varepsilon_{1}, \varepsilon_{2}$ be any two real numbers such that $\varepsilon_{1}>0$ and $\varepsilon_{2}>0$. Every planar graph with at least one edge has a $\left(\varepsilon_{1}, \varepsilon_{2}\right)$-Delaunay drawing.

The discussion of this section is summarized in the following theorem, which establishes that one can compute a drawing that approximates a Delaunay drawing for every planar graph, provided that the Delaunay disks are scaled down for the edges and are scaled up for the non-adjacent pairs of vertices by any arbitrarily small chosen amount.

Theorem 5. Let $G$ be an embedded planar graph. For any given values of $\varepsilon_{1}, \varepsilon_{2}$ such that $\varepsilon_{1}>0$ and $\varepsilon_{2}>0, G$ admits an embedding preserving $\left(\varepsilon_{1}, \varepsilon_{2}\right)$-Delaunay drawing. Also, there exist embedded planar graphs that do not have an embedding preserving $\left(0, \varepsilon_{2}\right)$-Delaunay drawing and embedded planar graphs that do not have an embedding preserving $\left(\varepsilon_{1}, 0\right)$-Delaunay drawing.

\section{Conclusions and Open Problems}

In this paper we have introduced an approximate version of the well-studied proximity drawings. In comparison with the standard definition of region of influence based proximity drawing, our drawings consider a slightly smaller region of influence for the adjacent pairs of vertices and a slightly larger region for the non-adjacent pairs. The amount by which the region of influence can be scaled up or down depends on two non-negative real numbers $\varepsilon_{1}$ and $\varepsilon_{2}$; the resulting straight-line drawing is called an $\left(\varepsilon_{1}, \varepsilon_{2}\right)$-proximity drawing. Intuitively, the smaller these parameters are the closer an $\left(\varepsilon_{1}, \varepsilon_{2}\right)$-proximity drawing is to the standard proximity drawing.

The paper has investigated the approximation of three well-known proximity drawings, namely Gabriel drawings, Delaunay drawings, and $\beta$-drawings. For each of these types of proximity drawings, we showed that every planar graph has a planar straightline drawing that can be made arbitrarily close to satisfy the usual proximity rule. This contrasts with well-known results that only restricted subfamilies of planar graphs have a (standard) Gabriel drawing, or a Delaunay drawing, or a $\beta$-drawing. Also extremal cases which generalize and extend the notion of weak proximity have been investigated. A first natural direction for future research is therefore the following.

Question 1. Extend the study of approximate proximity to other classical or emerging families of proximity drawings, such as the rectangle of influence drawings and/or the witness Delaunay drawings. A good starting point for this question may be, for example [3].

We remark that the major contribution of this paper is in analyzing to what extent the class of representable graphs can vary if the standard definition of proximity is approximate in the manner described above. Based on the presented results, we believe that the proposed definition of approximate proximity may be effectively adopted in practice to represent planar graphs where proximity constraints need to be maintained. However, in order to do so, relevant questions about the area and the bit complexity of the computed drawings must be addressed. 
As an example, we recall a recent paper by Angelini et al. [1] proving that drawing a tree of maximum degree five as a Euclidean minimum spanning tree may require exponential area. Since the family of $\beta$-drawable trees for $\beta=2$ is the family of trees having maximum degree five and since a 2-drawing of a tree is also a Euclidean minimum spanning tree, it follows that $(0,0)-2$ drawings may require exponential area. On the other hand, every straight-line planar drawing is an $\left(\varepsilon_{1}, \varepsilon_{2}\right)$-proximity drawing for a sufficiently small value of $\varepsilon_{1}$ and a sufficiently large value of $\varepsilon_{2}$. In fact, every planar graph has a $(0, \infty)$-proximity drawing with integer coordinates and in polynomial area (see, e.g., [5]). This discussion leads to the following research direction.

Question 2. Study polynomial area approximation schemes, that is, for any fixed $\varepsilon_{1}$, $\varepsilon_{2}$, the size of the computed drawing is bounded by a polynomial in the number of vertices of the given graph. Similar studies have been described in [10] in the context of drawing a tree as a minimum spanning tree approximation.

Acknowledgments. This research was initiated during the Bici BWGD 2011: Bertinoro Workshop on Graph Drawing. The authors are thankful to the workshoppers for useful discussions and also to the anonymous referees for their helpful comments. Research supported in part by MIUR of Italy under project AlgoDEEP prot. 2008TFBWL4 and NSERC of Canada, as well as by EUROGIGA project GraDR 10-EUROGIGA-OP-003.

\section{References}

1. Angelini, P., Bruckdorfer, T., Chiesa, M., Frati, F., Kaufmann, M., Squarcella, C.: On the Area Requirements of Euclidean Minimum Spanning Trees. In: Dehne, F., Iacono, J., Sack, J.-R. (eds.) WADS 2011. LNCS, vol. 6844, pp. 25-36. Springer, Heidelberg (2011)

2. Aronov, B., Dulieu, M., Hurtado, F.: Witness (Delaunay) graphs. Comput. Geom. 44(6-7), 329-344 (2011)

3. Aronov, B., Dulieu, M., Hurtado, F.: Witness Rectangle Graphs. In: Dehne, F., Iacono, J., Sack, J.-R. (eds.) WADS 2011. LNCS, vol. 6844, pp. 73-85. Springer, Heidelberg (2011)

4. Bose, P., Lenhart, W., Liotta, G.: Characterizing proximity trees. Algorithmica 16(1), 83-110 (1996)

5. de Fraysseix, H., Pach, J., Pollack, R.: How to draw a planar graph on a grid. Combinatorica 10(1), 41-51 (1990)

6. Di Battista, G., Lenhart, W., Liotta, G.: Proximity Drawability: a Survey. In: Tamassia, R., Tollis, I.G. (eds.) GD 1994. LNCS, vol. 894, pp. 328-339. Springer, Heidelberg (1995)

7. Di Battista, G., Liotta, G., Whitesides, S.: The strength of weak proximity. J. Discrete Algorithms 4(3), 384-400 (2006)

8. Dillencourt, M.B.: Realizability of Delaunay triangulations. Inf. Process. Lett. 33(6), 283287 (1990)

9. Dillencourt, M.B., Smith, W.D.: A Simple Method for Resolving Degeneracies in Delaunay Triangulations. In: Lingas, A., Carlsson, S., Karlsson, R. (eds.) ICALP 1993. LNCS, vol. 700, pp. 177-188. Springer, Heidelberg (1993)

10. Di Giacomo, E., Didimo, W., Liotta, G., Meijer, H.: Drawing a Tree as a Minimum Spanning Tree Approximation. In: Cheong, O., Chwa, K.-Y., Park, K. (eds.) ISAAC 2010, Part II. LNCS, vol. 6507, pp. 61-72. Springer, Heidelberg (2010) 
11. Jaromczyk, J.W., Toussaint, G.T.: Relative neighborhood graphs and their relatives. Proc. IEEE 80(9), 1502-1517 (1992)

12. Lenhart, W., Liotta, G.: Proximity Drawings of Outerplanar Graphs. In: North, S.C. (ed.) GD 1996. LNCS, vol. 1190, pp. 286-302. Springer, Heidelberg (1997)

13. Li, X.: Applications of computational geometry in wireless networks. In: Cheng, X., Huang, X., Du, D.-Z. (eds.) Ad Hoc Wireless Networking, pp. 197-264. Kluwer Academic Publishers (2004)

14. Liotta, G.: Proximity drawings. In: Tamassia, R. (ed.) Handbook of Graph Drawing and Visualization. CRC Press (to appear) 